\title{
Dual Function of Pneumolysin in the Early Pathogenesis of Murine Pneumococcal Pneumonia
}

\author{
Jeffrey B. Rubins, „\$ Darlene Charboneau, * James C. Paton," Timothy J. Mitchell, ' Peter W. Andrew, \\ and Edward N. Janoff ${ }^{\text {ts }}$ \\ * Pulmonary Disease Division and ${ }^{\ddagger}$ Infectious Disease Division, Department of Medicine, Veterans Affairs Medical Center, and \\ 'Department of Medicine, University of Minnesota School of Medicine, Minneapolis, Minnesota 55417; "Department of Microbiology, \\ Women's and Children's Hospital, Adelaide, Australia; and 'Department of Microbiology and Immunology, University of Leicester, \\ Leicester, LE1-9HN, United Kingdom
}

\begin{abstract}
Streptococcus pneumoniae is one of the most common etiologic agents of community-acquired pneumonia, particularly bacteremic pneumonia. Pneumolysin, a multifunctional cytotoxin, is a putative virulence factor for $S$. pneumoniae; however, a direct role for pneumolysin in the early pathogenesis of pneumococcal pneumonia has not been confirmed in vivo. We compared the growth of a pneumolysindeficient (PLY $[-])$ type 2 S. pneumoniae strain with its isogenic wild-type strain (PLY $[+]$ ) after direct endotracheal instillation of bacteria into murine lungs. Compared with PLY ( - ) bacteria, infection with PLY (+) bacteria produced greater injury to the alveolar-capillary barrier, as assayed by albumin concentrations in alveolar lavage, and substantially greater numbers of $\operatorname{PLY}(+)$ bacteria were recovered in alveolar lavages and lung homogenates at 3 and $6 \mathrm{~h}$ after infection. The presence of pneumolysin also contributed to the development of bacteremia, which was detected at $3 \mathrm{~h}$ after intratracheal instillation of PLY $(+)$ bacteria.
\end{abstract}

The direct effects of pneumolysin on lung injury and on the ability of pneumococci to evade local lung defenses was confirmed by addition of purified recombinant pneumolysin to inocula of PLY $(-)$ pneumococci, which promoted growth of PLY $(-)$ bacteria in the lung to levels comparable to those seen with the PLY $(+)$ strain. We further demonstrated the contributions of both the cytolytic and the complement-activating properties of pneumolysin on enhanced bacterial growth in murine lungs using genetically modified pneumolysin congeners and genetically complement-deficient mice.

Thus, pneumolysin facilitates intraalveolar replication of pneumococci, penetration of bacteria from alveoli into the interstitium of the lung, and dissemination of pneumococci into the bloodstream during experimental pneumonia. Moreover, both the cytotoxic and the complement-activating activities of pneumolysin may contribute independently to the acute pulmonary injury and the high rates of bacteremia which characterize pneumococcal pneumonia. (J. Clin. In-

Address correspondence to J. B. Rubins, M.D., Pulmonary (111N), Minneapolis VA Medical Center, One Veterans Drive, Minneapolis, MN 55417.

Received for publication 17 May 1994 and in revised form 15 September 1994.

The Journal of Clinical Investigation, Inc.

Volume 95, January 1995, 142-150 vest. 1995.95:142-150.) Key words: Streptococcus pneumoniae $\cdot$ streptolysin $\cdot$ complement $\cdot$ mice $\bullet$ lung

\section{Introduction}

Pneumococcal pneumonia is associated with high rates of invasive disease and the attendant early mortality which accompanies bacteremic infections (1-3). Pneumolysin, a major pneumococcal cytotoxin, is a putative virulence factor in Streptococcus pneumoniae infection. Pneumolysin is a potent cytotoxin that injures immune $(4,5)$ and respiratory cells in vitro $(6-8)$. Instillation of pneumolysin into murine lungs reproduces many of the histological findings of pneumococcal pneumonia (9), perhaps through its ability to directly activate the classical complement system (10) and cellular phospholipase A (Rubins, J. B., manuscript submitted for publication), and stimulate cytokine release from monocytes (11). Moreover, genetically designed functionally pneumolysin-deficient (PLY $[-])^{1}$ mutant pneumococcal strains have been demonstrated to be less virulent than isogenic wild-type (PLY $[+]$ ) strains after intraperitoneal or intranasal injection in mice $(12,13)$. Also, immunization with pneumolysin protects animals by delaying or preventing death after challenge with virulent pneumococci $(14,15)$.

However, a direct role of pneumolysin in the early pathogenesis of pneumococcal pneumonia has not been completely defined in vivo. It is not known whether the amounts of pneumolysin required to produce cytolytic and pro-inflammatory effects in vitro are actually released by pneumococci during infection in vivo. Also, pneumolysin is known to be readily inactivated by inhibitors which may be present in the pulmonary alveolus, such as oxidants including hydrogen peroxide produced by the pneumococcus itself (16), and by cholesterol (17) derived from surfactant, cellular membranes, and serum lipoproteins (18).

We now report that pneumolysin increases bacterial multiplication in the lung and facilitates tissue invasion and bacteremia during the initial phase of murine pneumococcal pneumonia. In addition, we show that the cytotoxicity and the complement-activating activities of pneumolysin appear to have distinct roles in supporting bacterial replication and invasion during experimental pneumonia.

\section{Methods}

Bacterial strains and preparation of inocula. A PLY(-) mutant type $2 S$. pneumoniae strain (PLN-A) was previously constructed from strain

1. Abbreviations used in this paper: e.t., endotracheal; $\mathrm{HU}$, hemolytic units; $\mathrm{LD}_{50}, 50 \%$ lethal dose; PLY $(+)$ and $(-)$, pneumolysin-sufficient and -deficient. 
D39 by insertion-duplication mutagenesis as described (12). The mutant PLN-A strain produced a type 2 capsule as characterized by Quellung reaction using antiserum obtained from Statens Seruminstitut (Copenhagen, Denmark), and the size of the capsule was indistinguishable from that of the parenteral D39 strain on blood agar plates. PLY (+) wild-type and PLY $(-)$ mutant strains were stored at $-70^{\circ} \mathrm{C}$ on glass beads in trypticase soy broth supplemented with $10 \%$ glycerol, and were passed through mice immediately before experiments to ensure virulence. Mutant strains were selected on erythromycin plates as described (12). Bacteria were grown to mid-logarithmic phase under micro aerobic conditions in brain-heart infusion broth (DIFCO Laboratories, Detroit, MI), washed, and then resuspended in Dulbecco's PBS for inoculation. Inoculum size was confirmed by quantitative culture on blood agar plates (trypticase soy agar plates containing 5\% sheep erythrocytes, GIBCO BRL, Gaithersburg, MD).

Animals. Specific pathogen-free white female National Institutes of Health Swiss outbred mice (20-30 g) were obtained from Harlan Sprague-Dawley, Inc. (Indianapolis, IN). Inbred B10.D2/oSn mice genetically deficient in the fifth component of complement (C5-) and the congenic wild-type B10.D2/nSn mice (C5+) were procured from Jackson Laboratories (Bar Harbor, ME) (19). Animals were housed in a pathogen-free barrier facility fully accredited by the American Association for the Accreditation of Laboratory Animal Care. Animal studies were performed in accordance with the guidelines established in the NIH "Guide for the Care and Use of Laboratory Animals" (Department of Health, Education and Welfare Publication No. [NIH] 85-23, Office of Science and Health Reports, Division of Research Resources, Bethesda, MD), and the research was approved by the Animal Study Subcommittee of the Minneapolis Veterans Affairs Medical Center (Minneapolis, MN).

Purification of recombinant pneumolysins. Recombinant pneumolysins congeners, modified to have either reduced cytolytic activity (Trp $433>$ Phe) or reduced ability to activate complement (Asp 385 $>$ Asn), were produced by single-point site-directed mutagenesis as described $(20,21)$. Modified and wild-type recombinant pneumolysins were purified from lysates of Escherichia coli JM109 harboring the pneumolysin gene in the expression vector pKK233-2 by hydrophobic chromatography on a TSK phenyl-5PW high pressure liquid chromatography column (22). Purified recombinant pneumolysin migrated as a single band with approximate molecular mass of $53 \mathrm{kD}$ on silver-stained SDS-polyacrylamide gels. Wild-type recombinant pneumolysin had a specific activity of $3 \times 10^{5}$ hemolytic units (HU) per mg protein, assayed as described (7).

Endotracheal instillation of bacterial inocula. After inducing anesthesia by i.p. injection of $50 \mathrm{mg} / \mathrm{kg}$ sodium pentobarbital, mice were suspended vertically by supporting the lower incisor teeth on a wire loop and retaining the upper incisors with a rubber band as described (23). While using a small retractor to displace the tongue anteriorly, the oropharynx was transilluminated and under direct visualization the trachea was cannulated with a blunt-tipped 22 gauge metal needle attached to a microliter syringe. During inspiration, the bacterial suspension in PBS ( $50 \mu \mathrm{l})$ followed by an equal volume of air was injected, and the animals were maintained upright at a $45^{\circ}$ angle until fully recovered from anesthesia $(\sim 30 \mathrm{~min})$. This technique reproducibly delivered greater than $99 \%$ of the inoculum to the lungs, as determined by quantitative culture of lungs immediately after infection, and equally distributed the inocula to both lower lobes, as determined by endotracheal (e.t.) instillation of India ink.

Determination of $50 \%$ lethal dose after i.p. and e.t. infection. Groups of 4-6 mice were infected with increasing concentrations of PLY $(+)$ and PLY $(-)$ bacteria by either i.p. or e.t. injection. Survival was recorded at 8-h intervals for $96 \mathrm{~h}$, and the $50 \%$ lethal dose $\left(L D_{50}\right)$ was calculated by the method of Reed and Muench (24).

Recovery of bacteria from blood and lung. Mice were sacrificed by cervical dislocation at selected times after infection. After opening the thorax, $100 \mu \mathrm{l}$ of blood for culture was obtained by cardiac puncture. The trachea was then surgically exposed and cannulated, and the lungs were lavaged with $1 \mathrm{ml}$ of PBS. Approximately $75 \%$ of the lavage fluid was collected by aspiration after several seconds. This single-bolus technique reproducibly recovered $>95 \%$ of the instilled inoculum, as assessed by quantitative culture immediately after instillation, and had the advantage of decreasing transudation of serum proteins into the lavage, which might occur during repetitive lavage. After removal of an aliquot for quantitative culture, the lavage was cleared of cells by centrifugation and frozen at $-20^{\circ} \mathrm{C}$.

After lavage, the lungs were dissected from major vessels and bronchi, rinsed, and homogenized in $2 \mathrm{ml}$ of sterile PBS in a glass tissue grinder. The number of viable bacteria in samples of blood, alveolar lavage, and lung homogenate was determined by quantitative culture of serial dilutions on blood agar plates. Bacteria were identified as pneumococci by colony morphology of $\alpha$-hemolytic organisms and by Optochin sensitivity, and PLY (-) bacteria were identified by growth on erythromycin plates and by lack of hemolysin activity. The total number of bacteria in each murine lung was calculated as the sum of the total number of bacteria in alveolar lavage plus lung homogenate samples.

Determination of albumin concentration in lavage. The concentration of albumin in lavage supernatants was measured as an index of alveolar-capillary barrier disruption. Lavage proteins (40- $\mu$ l aliquots) and albumin standards were separated by $10 \%$ SDS-polyacrylamide gel electrophoresis. After Coomassie staining, gels were analyzed by video densitometry (model 6́20; Bio-Rad Laboratories, Hercules, CA), and the concentration of albumin determined by comparison to standards.

Assay of endotoxin activity. Endotoxin activities in recombinant pneumolysin preparations were quantified using a Limulus amoebocyte lysate test (Pyrotell; Association of Cape Cod, Woods Hole, MA).

Statistics. Geometric means and standard errors of means (SE) of bacterial colony forming units (CFU) were calculated on log-transformed data, and then data was converted by exponential transformation to CFU. Where indicated, statistical significance was calculated by comparison of geometric means by unpaired two-tailed $t$-test. Each datum point in figures and tables represents the mean $\pm S E$ of 3-4 animals, and results of each experiment were confirmed by at least one repeat experiment.

\section{Results}

Relative virulence of $P L Y(+)$ wild-type and PLY(-) mutant strains by systemic and mucosal infection. After systemic infection by i.p. infection, the type $2 S$. pneumoniae PLY $(+)$ strain was highly virulent, with an $\mathrm{LD}_{50}$ of $25 \mathrm{CFU}$. In contrast, the PLY (-) mutant strain was substantially less virulent, with an $\mathrm{LD}_{50}$ i.p. dose of $500 \mathrm{CFU}$. Furthermore, death was appreciably delayed in the groups infected with the PLY $(-)$ mutant compared with the PLY $(+)$ strain (median survival times 72 and $20 \mathrm{~h}$, respectively).

The greater virulence of the PLY(+) wild-type compared with the PLY $(-)$ mutant pneumococcal strain with systemic infection paralleled that with mucosal infection by e.t. instillation. However, mice showed a marked resistance to pneumococcal infection by mucosal compared to systemic infection. The $\mathrm{LD}_{50}$ for e.t. infection with PLY $(-)$ bacteria was again one $\log$ higher than that for PLY $(+)$ bacteria $\left(10^{8}\right.$ CFU versus $10^{7}$ CFU), but each was five logs greater than the corresponding i.p. doses. In summary, the PLY $(-)$ mutant type $2 S$. pneumoniae strain was less virulent than the isogenic wild-type strain after both routes of infection, consistent with previous observations (12). Moreover, the mouse lung appeared to be capable of clearing extraordinary numbers of virulent bacteria, suggesting that it serves as a major line of defense against disseminated pneumococcal infection. 


\begin{tabular}{|c|c|c|c|c|}
\hline \multirow[b]{3}{*}{ Inoculum } & \multicolumn{4}{|c|}{ Albumin concentration $(\mathrm{mg} / \mathrm{ml})$} \\
\hline & \multicolumn{4}{|c|}{ Time after infection (h) } \\
\hline & 0 & 3 & 6 & 24 \\
\hline PBS & $.06 \pm .01$ & $.09 \pm .03$ & $.18 \pm .07$ & $.22 \pm .05$ \\
\hline Pneumolysin (5 HU) & $.07 \pm .02$ & $.32 \pm .14^{8}$ & $.42 \pm .12^{8}$ & $.34 \pm .16$ \\
\hline PLY(+) strain* & $.07 \pm .01$ & $.34 \pm .11^{8}$ & $.72 \pm .14^{8 \|}$ & $.37 \pm .12$ \\
\hline PLY(-) strain ${ }^{\ddagger}$ & & $.12 \pm .07$ & $.18 \pm .04$ & $.22 \pm .01$ \\
\hline$+5 \mathrm{HU}$ pneumolysin & & $.52 \pm 0.8^{8 \|}$ & $.46 \pm .34$ & $.56 \pm .39$ \\
\hline$+50 \mathrm{HU}$ pneumolysin & & $1.12 \pm .26^{3 * *}$ & $1.35 \pm .32^{1 * *}$ & $1.22 \pm .34^{9 * *}$ \\
\hline
\end{tabular}

${ }^{*}$ Inocula $=5 \times 10^{6} \mathrm{CFU}$ in $0.05 \mathrm{ml}$ PBS. Data represent mean $\pm \mathrm{SE}$ of three animals. ${ }^{\ddagger}$ Inocula $=1.35 \times 10^{7} \mathrm{CFU}$ in $0.05 \mathrm{ml} \mathrm{PBS}$. Mean $\pm \mathrm{SE}$, $n=3$. ${ }^{8} P<.02$ compared with PBS; $\| P<.02$ compared wit PLY(-) strain; $\quad{ }^{\prime} P<.05$ compared with PBS; $* * P<.05$ compared with PLY(-) strain.

Comparison of recovery of $P L Y(+)$ and $P L Y(-)$ strains from lung and blood. We next investigated whether pneumolysin might disrupt the protective function of the lung in vivo. Purified pneumolysin injures alveolar epithelial cells in vitro and the alveolar-capillary barrier in isolated perfused murine lungs (7). We have speculated that pneumolysin injury to the alveolar epithelium may produce alveolar flooding with serous exudate, which may provide necessary nutrients and promote rapid multiplication of pneumococci within the alveoli. To test this hypothesis in vivo, mice were inoculated e.t. with either the wild-type PLY $(+)$ type 2 strain $\left(5 \times 10^{6} \mathrm{CFU}\right)$ or the PLY (-) mutant strain $\left(1.35 \times 10^{7} \mathrm{CFU}\right)$. At selected times after infection, mice were sacrificed and samples of alveolar lavage, lung homogenates, and blood were obtained for culture and biochemical studies. Based on previous histological studies (25), times were selected to approximate the period before significant neutrophil influx (congestion, $3 \mathrm{~h}$ ), the acute phase of neutrophil influx (red hepatization, $6 \mathrm{~h}$ ), and the period of established neutrophil response (gray hepatization, $24 \mathrm{~h}$ ).

To determine whether pneumolysin released from bacteria was actively cytotoxic in vivo, we assayed albumin concentrations in the alveolar lavage samples as a marker of alveolarcapillary injury. Infection with PLY $(+)$ bacteria was associated with a 3.7-fold increase in lavage albumin at $3 \mathrm{~h}$ and a fourfold increase at $6 \mathrm{~h}$ compared with control mice receiving PBS e.t. (Table I). In contrast, no significant increase in albumin was detected in lavage from mice infected with the PLY $(-)$ strain as compared to PBS-treated mice. Therefore, PLY $(+)$ bacteria, but not PLY (-) bacteria, appeared to induce a degree of mucosal injury sufficient to disrupt the alveolar-capillary barrier in murine lungs in vivo.

Corresponding to the greater alveolar injury produced by the PLY $(+)$ bacteria, significantly greater numbers of bacteria were recovered from murine lungs infected with PLY $(+)$ pneumococci at 3 and $6 \mathrm{~h}$ after infection, compared with those infected with PLY(-) mutants (Fig. 1). Recovery of PLY(+) bacteria from lungs was more than threefold higher at $3 \mathrm{~h}$ and 30-fold higher at $6 \mathrm{~h}$ compared to PLY $(-)$ bacteria. However, despite the reported cytotoxicity of pneumolysin to immune leukocytes $(4,5)$, the rate of net clearance of both PLY $(+)$ and PLY (-) bacteria from 6 to $24 \mathrm{~h}$ after infection was very similar ( 14.5 and $13 \%$ per hour, respectively).
The increased recovery of viable PLY $(+)$ bacteria from murine lungs during early infection reflected the markedly greater numbers of PLY $(+)$ bacteria recovered from both alveolar lavages and lung homogenates at 3 and $6 \mathrm{~h}$ after infection, compared with the PLY (-) strain (Fig. 2). There was a striking dissociation between the numbers of organisms in the alveolar and tissue samples at $6 \mathrm{~h}$ after infection with PLY $(+)$ bacteria (Fig. $2 A$ ), suggesting increased penetration of pneumococci from pulmonary alveoli into interstitium. In contrast, numbers of PLY (-) pneumococci in alveolar lavage did not increase at any time point, nor did they show any significant invasion into lung tissue (Fig. $2 B$ ). Rather, numbers of PLY $(-)$ bacteria continued to decrease in both compartments after $3 \mathrm{~h}$.



Figure 1. Recovery of pneumococci from murine lungs. Total number of bacteria in murine lungs were calculated from quantitative culture of alveolar lavages and lung homogenates obtained at the indicated times after e.t. instillation of a pneumolysin-deficient mutant type 2 strain (O) or the isogenic wild-type strain (๑). Each datum point represents the geometric mean $\pm S E$ of the total CFU per lung for three animals. Comparison of geometric mean CFU for PLY(+) and PLY(-) strains by unpaired $t$-test; $*, P<.05 ; * *, P<.01$. 
A.

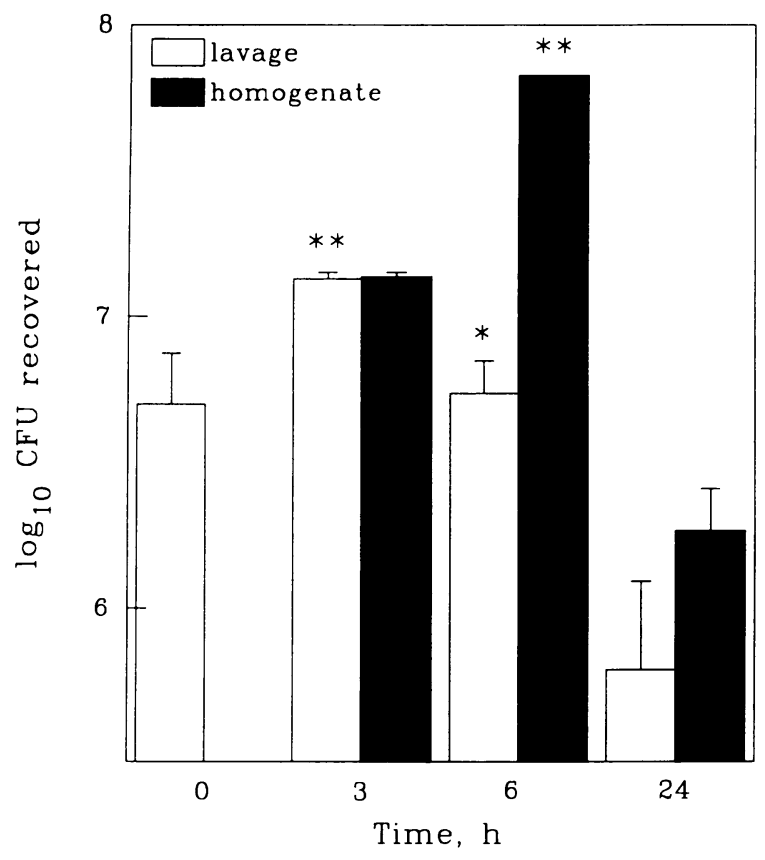

B.

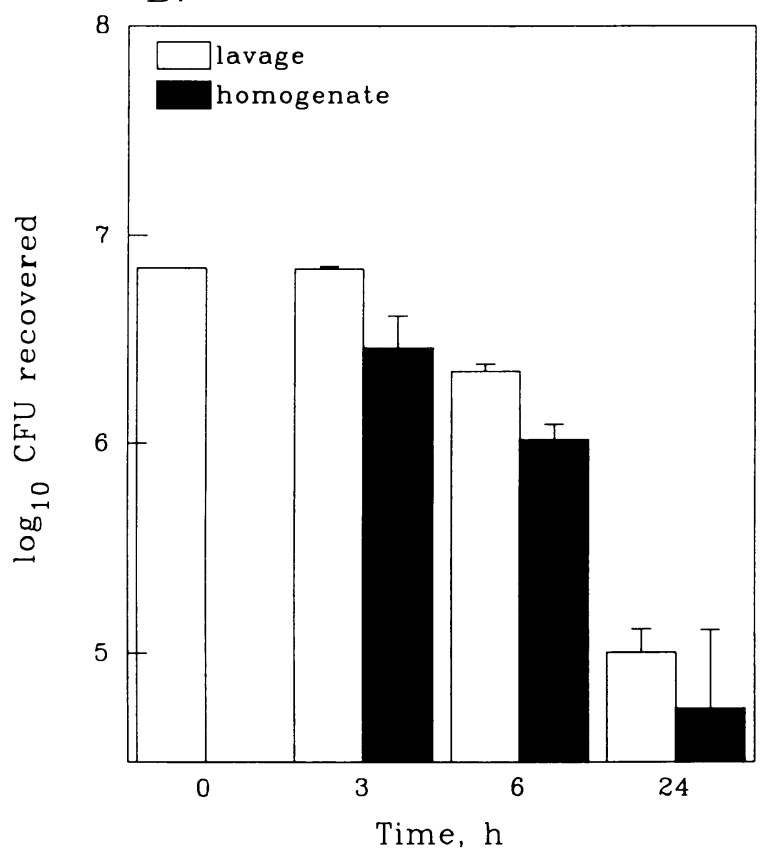

Figure 2. Recovery of pneumococci from alveolar lavages and lung homogenates. Samples of alveolar lavage ( .7$)$ and lung homogenates $(\boldsymbol{\square})$ were quantitatively cultured at the indicated intervals after infection with $(A) \operatorname{PLY}(+)$ and $(B)$ PLY $(-)$ strains. Datum points and statistical comparisons are as described for Fig. 1.

Consistent with the increased invasion of PLY $(+)$ bacteria from alveoli into lung tissue. PLY $(+)$ bacteria also produced earlier and higher grade bacteremia than did PLY $(-)$ bacteria

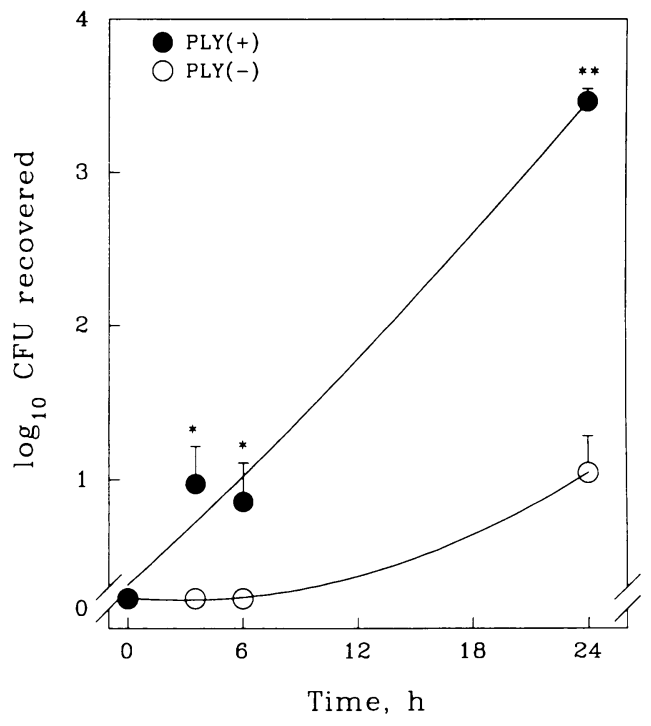

Figure 3. Recovery of pneumococci from blood after endotracheal instillation. Blood samples were collected for quantitative culture at the indicated times after e.t. instillation of $\operatorname{PLY}(-)(c)$ and $\operatorname{PLY}(+)(\bullet)$ strains. Each datum point represents the geometric mean \pm SE for three animals. Datum points below the axis break represent 0 bacteria. Data is representative of two experiments. Statistical comparisons are as described for Fig. 1.

(Fig. 3 ). PLY ( + ) bacteria appeared in blood at 3 and $6 \mathrm{~h}$. and numbers dramatically increased at $24 \mathrm{~h}$. PLY $(+)$ bacteremia increased to $10^{6}-10^{7} \mathrm{CFU} / \mathrm{ml}$ at $48 \mathrm{~h}$, and the animals began to die during the subsequent $24 \mathrm{~h}$. In contrast, PLY $(-)$ bacteria were not detected in blood before $24 \mathrm{~h}$, when more modest numbers of bacteria were recovered. Also, the number of PLY ( - ) bacteria in the blood at 48 and $72 \mathrm{~h}$ was highly variable between animals. A minority of animals who appeared overtly ill were bacteremic with $10^{6} \mathrm{CFU} / \mathrm{ml}$ at $72 \mathrm{~h}$, and some of these died during the next $48 \mathrm{~h}$. However, the majority of the mice infected with PLY $(-)$ appeared well and had no detectable bacteremia at $72 \mathrm{~h}$.

To ascertain whether the enhanced growth of PLY $(+)$ pneumococci in infected murine lungs reflected an intrinsic defect in the growth of the PLY $(-)$ mutants, we compared the in vitro growth of the two strains in several substrates. In contrast to growth in vivo, the PLY $(-)$ mutant strain growth in vitro when incubated in brain-heart infusion broth. in ToddHewitt media, and in homogenates of murine lung (doubling times of 32. 37. and $20 \mathrm{~min}$. respectively), was equal or greater than that of the PLY (+) strain (doubling times of 32, 37, and 22 min, respectively).

Taken together, these studies suggested that the presence of endogenous production of pneumolysin was associated with enhancement of specific pathogenic effects of $S$. pneumoniae infection. including increased intraalveolar replication of bacteria, penetration of organisms from alveoli into the interstitium of the lung, and dissemination of pneumococci into the bloodstream.

Addition of recombinant pneumolysin to inocula of $P L Y(-)$ bacteria. To confirm that differences in pneumolysin production accounted for the observed differences in specific pathogenic effects of the PLY (+) strain compared with the PLY( - ) strain, 


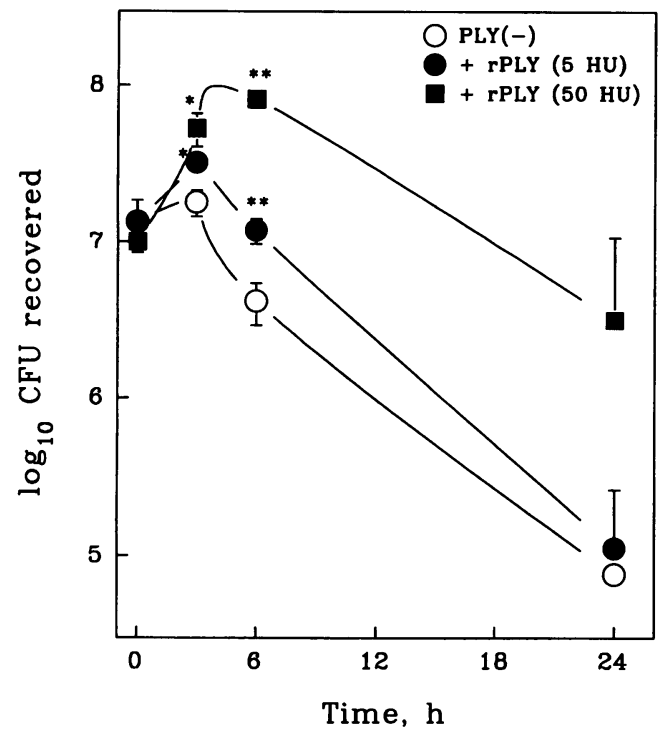

Figure 4. Effect of added pneumolysin on recovery of PLY $(-)$ bacteria from murine lungs. Total numbers of bacteria in murine lungs were determined from quantitative culture of alveolar lavage and lung homogenates obtained at the indicated intervals after e.t. instillation of inocula of PLY (-) bacteria, either alone (O) or supplemented with purified recombinant pneumolysin $(+r P L Y)$ at concentrations of $5 \mathrm{HU} / \mathrm{ml}(\bullet)$ or $50 \mathrm{HU} / \mathrm{ml}(\square)$. Datum points are as described for Fig. 1. Comparison of geometric mean CFU for PLY $(-) \pm \mathrm{rPLY}$ by unpaired $t$-test. ${ }^{*}, P$ $<.05, * *, P<.01$.

inocula of PLY(-) pneumococci $\left(10^{7} \mathrm{CFU}\right)$ were supplemented with either 5 or $50 \mathrm{HU} / \mathrm{ml}$ of purified recombinant pneumolysin. In preliminary studies, these concentrations of pneumolysin approximated the cytolytic activity released in vitro by $10^{7}$ and $10^{8} \mathrm{CFU} / \mathrm{ml}$, respectively, of the PLY (+) strain. Addition of recombinant pneumolysin to PLY $(-)$ pneumococci produced a dose-dependent increase in alveolar lavage albumin concentrations ( Table I), confirming injury to the alveolar-capillary barrier. Recombinant pneumolysin also induced a dose-dependent increase in recovery of PLY $(-)$ bacteria from murine lungs early in the course of infection, similar to PLY $(+)$ bacteria (Fig. 4). Addition of $50 \mathrm{HU} / \mathrm{ml}$ pneumolysin substantially increased total numbers of PLY $(-)$ bacteria recovered from lungs at 3 and $6 \mathrm{~h}$, approximating the effects observed with PLY $(+)$ bacteria.

To control for the effects of endotoxin contamination of recombinant pneumolysin, we added of $15 \mathrm{pg} / \mathrm{ml}$ endotoxin, equivalent to the contaminant concentrations measured by Limulus lysate assay in the pneumolysin preparations, to PLY (-) bacteria. These suspensions neither caused albumin influx into alveolar lavage, nor increased numbers of bacteria in lavage, homogenate or blood (data not shown). Thus, endotoxin impurities did not account for the pathogenic effects of pneumolysin in this model.

Role of cytolytic activity in pneumolysin-facilitated bacterial growth. In addition to its potent cytolytic activity, pneumolysin is also capable of activating the classical complement system (10). Molecular studies have defined distinct peptide sequences for the cytolytic and complement-stimulating active sites $(20,21)$. Furthermore, the functional independence of these two activities has been demonstrated by the persistence

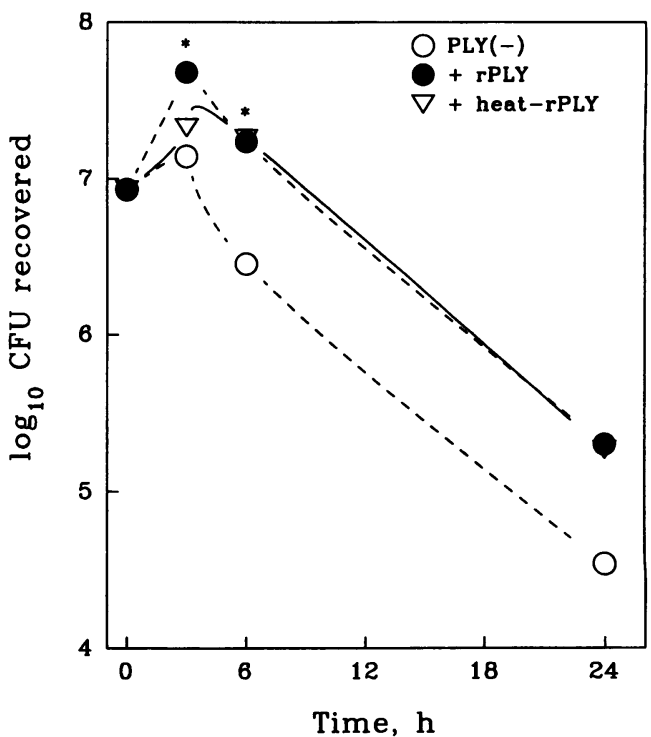

Figure 5. Effect of heat-inactivated pneumolysin on recovery of PLY ( - ) bacteria from murine lungs. Total numbers of bacteria recovered from murine lungs were determined at the indicated times after e.t. instillation of inocula of PLY $(-)$ bacteria, either alone $(O)$ or supplemented with untreated recombinant pneumolysin (rPLY, $10 \mathrm{HU} / \mathrm{ml} ; \bullet$ ) or heat-inactivated pneumolysin (heat-rPLY, $\triangle$ ). Datum points and statistical comparisons are as described for Fig. 4.

of complement activation after inhibition of pneumolysin cytotoxicity (10). To examine the contributions of these two activities on pneumolysin-facilitated PLY $(-)$ bacterial growth, we first inactivated cytolytic activity by incubating recombinant pneumolysin at $56^{\circ} \mathrm{C}$ for 30 min before addition to inocula of PLY (-) bacteria. Hemolytic activity was reduced by $>99 \%$ after heat inactivation (6). In comparison with untreated pneumolysin $(10 \mathrm{HU} / \mathrm{ml})$, heat-inactivated pneumolysin had less effect on recovery of PLY $(-)$ bacteria from lungs at $3 \mathrm{~h}$ (Fig. $5)$. However, addition of heat-inactivated pneumolysin significantly increased numbers of PLY $(-)$ bacteria at $6 \mathrm{~h}$, identical to the effect of untreated pneumolysin. This increased recovery of PLY $(-)$ bacteria at $6 \mathrm{~h}$ reflected a significant persistence of bacterial inoculum in alveolar lavage and increased tissue invasion (data not shown).

Thus, the addition of exogenous purified recombinant pneumolysin to PLY $(-)$ bacteria reproduced many of the sequential changes in bacterial growth and invasion seen with pneumolysin-producing organisms. However, in this in vivo model, the activity of pneumolysin was related only in part to its heatlabile cytolytic activity, suggesting that an additional property of pneumolysin might be involved in these effects.

Role of complement activation in pneumolysin-facilitated bacterial growth. Because the complement-activating activity of pneumolysin can persist after heat-inactivation of cytotoxicity (Mitchell, T. J., unpublished data), we hypothesized that the persistent effects of heat-inactivated pneumolysin on bacterial growth might be related to its ability to activate complement. To test this hypothesis, we added modified recombinant pneumolysins to e.t. inocula of PLY $(-)$ bacteria in our murine model. We used two modified pneumolysins with single amino acid substitutions generated by site-directed mutagenesis; one 
with full cytolytic activity but reduced ability to activate complement (Asp $385>$ Asn, complement deficient), and the other with reduced cytolytic activity $\left(10^{3} \mathrm{HU} / \mathrm{mg}\right.$, representing $0.33 \%$ of wild-type toxin activity) but normal complement-activating activity (Trp $433>$ Phe, cytolytic deficient) $(20,21)$.

After e.t. infection, recovery of PLY $(-)$ bacteria from murine lungs at $3 \mathrm{~h}$ was appreciably increased after addition of $1 \mu \mathrm{g} / \mathrm{ml}$ (equivalent to $15 \mathrm{HU}$ of unmodified toxin) of the complement-deficient pneumolysin possessing full cytolytic activity (Fig. $6 \mathrm{~A}$ ), whereas addition of the "cytolytic-deficient" pneumolysin produced no increase (Fig. $6 \mathrm{~B}$ ). Conversely, recovery of PLY $(-)$ bacteria at $6 \mathrm{~h}$ was not increased after addition of the complement-deficient pneumolysin, but was appreciably increased after addition of cytolytic-deficient pneumolysin having normal complement-stimulating activity. Similar to the effects of heat-inactivated pneumolysin, the increased number of PLY $(-)$ bacteria at $6 \mathrm{~h}$ reflected an appreciable persistence of bacterial inoculum, predominantly in alveolar lavage samples, in mice receiving pneumolysin with intact complementactivating activity (data not shown).

In summary, studies with recombinant pneumolysins suggested that pneumolysin's cytolytic activity accelerated bacterial growth and invasion into lung tissue during the initial period after infection, whereas its ability to activate complement appeared to facilitate bacterial growth in alveoli at later times.

Recovery of $P L Y(+)$ and $P L Y(-)$ bacteria from C5-deficient mice. Complement plays a critical role in the initial clearance of pneumococci from the lung by generating neutrophil chemotaxins (primarily C5a) and by opsonizing bacteria (C3a and C5a) (26). The ability of pneumolysin to activate complement has been speculated to subvert this essential host defense by diverting complement away from the bacteria, thereby compromising complement-mediated opsonization and phagocytosis. Therefore, we hypothesized that addition of pneumolysin possessing intact complement-activating properties to bacterial inocula might increase the recovery of PLY $(-)$ bacteria at $6 \mathrm{~h}$ by interfering with complement-mediated clearance by neutrophil phagocytosis. We further investigated this hypothesis using mice with a specific congenital complement deficiency, which have reduced pulmonary granulocyte recruitment in response to S. pneumoniae (19). Congenic C5-deficient (C5-) and C5sufficient $(\mathrm{C} 5+)$ mice were infected e.t. with PLY $(+)$ and PLY (-) bacteria, and samples of alveolar lavage, lung homogenates, and blood were obtained for culture and biochemical studies.

As observed with Swiss mice, PLY $(+)$ bacteria produced greater albumin influx into alveolar lavage of C5- mice at 3 and $6 \mathrm{~h}$ than did PLY $(-)$ (data not shown). Similarly, total numbers of PLY $(+)$ bacteria were increased lungs at 3 and 24 $\mathrm{h}$ after infection, compared with PLY(-) bacteria (Fig. 7). However, several important differences were observed with e.t. infection in C5- mice compared with C5+ mice. First, the total numbers of PLY $(-)$ and PLY $(+)$ bacteria in lungs were at least twofold greater at all times after e.t. infection of C5- mice compared with C5+ mice (Fig. 7). Second, earlier and higher grade bacteremia was seen in both PLY $(-)$ and PLY $(+)$ bacteria in C5- mice compared with complement-sufficient mice (data not shown). Third, the net clearance of PLY $(-)$ and especially PLY (+) mice at $24 \mathrm{~h}$ was substantially reduced in C5- mice (Fig. 7).
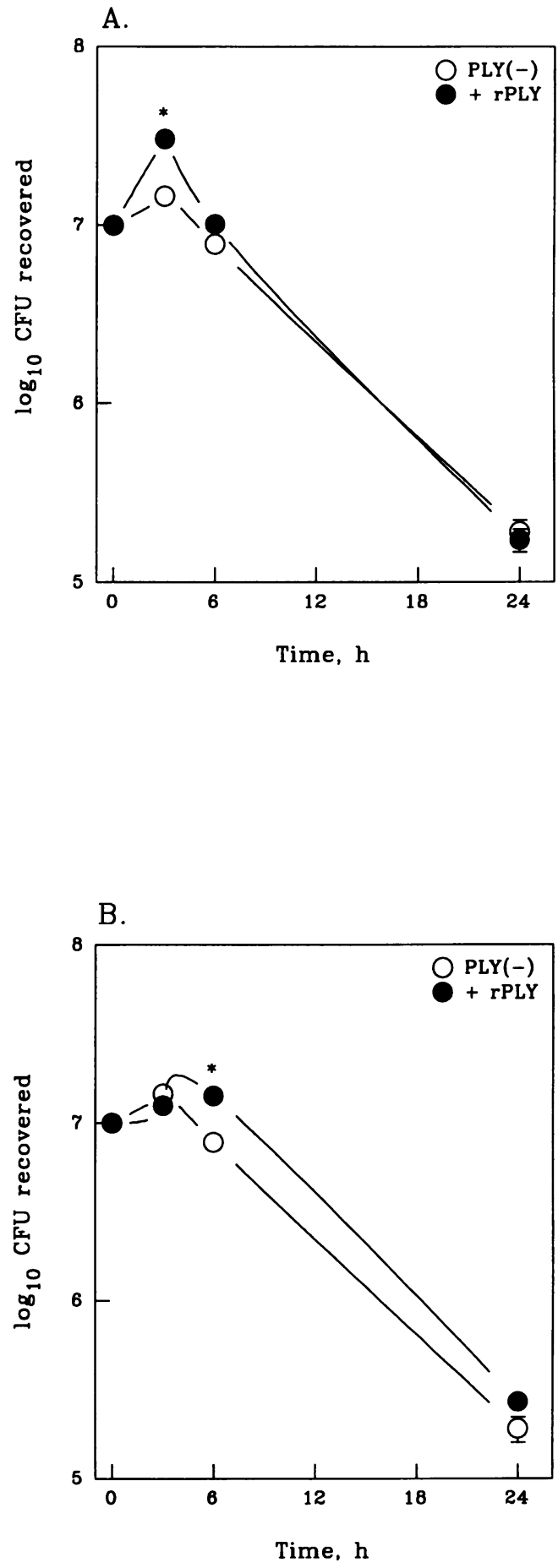

Figure 6. Effect of modified pneumolysins on recovery of PLY(-) bacteria from murine lungs. Total numbers of bacteria recovered from murine lungs were determined at the indicated times after e.t. instillation of inocula of PLY $(-)$ bacteria, either alone $(O)$ or supplemented with modified recombinant pneumolysins $(+\mathrm{rPLY}, 1 \mu \mathrm{g} / \mathrm{ml}, \bullet)$. Pneumolysins were genetically modified to have $(A)$ reduced ability to activate complement or $(B)$ reduced cytolytic activity. Each datum point represents the geometric mean \pm SE of total CFU per lung for four animals. Comparison of geometric mean CFU for PLY $(-) \pm$ rPLY by unpaired $t$-test. ${ }^{*}, P<.05$. 




Figure 7. Recovery of pneumococci from C5-deficient and C5-sufficient mice. Total bacterial CFU were determined at the indicated times after e.t. instillation of PLY $(-)$ bacteria $(O, \square)$ or PLY $(+)$ pneumococci $(\bullet, \square)$ in congenic C5-deficient $(O, \bullet)$ and C5-sufficient $(\square, \bullet)$ mice. Each datum point represents the geometric mean \pm SE of total CFU per lung for four animals. Statistical comparisons as are described for Fig. 1.

Finally, the total number of PLY $(-)$ bacteria appreciably increased from 3-6 h in C5- murine lungs (Fig. 7, open circles $)$, in contrast to the net clearance of PLY $(-)$ bacteria after $3 \mathrm{~h}$ consistently observed in C5+ mice (Fig. 7, open squares). In particular, net clearance of PLY $(-)$ bacteria from alveolar lavage at $6 \mathrm{~h}$ was markedly reduced in C5- mice (data not shown). Thus, a similar increase in numbers of PLY $(-)$ pneumococci at $6 \mathrm{~h}$ was seen when the complement system was manipulated, either by genetic deficiency of $\mathrm{C} 5$ or by addition of recombinant pneumolysins with intact complement-activating function. Furthermore, inhibition of complement appeared to predominantly decrease the net clearance of pneumococci from alveoli.

\section{Discussion}

Our studies provide in vivo evidence for the important role of pneumolysin in the early pathogenesis of pneumococcal pneumonia. Although pneumolysin's cytotoxicity to lung cells and its ability to disrupt the alveolar-capillary barrier has been previously established in vitro (7), the relevance of these observations to the pathogenesis of pneumococcal pneumonia in vivo has remained unknown for several reasons. First, because pneumolysin is an intracellular protein that is only released upon autolysis of the bacterium (27), it was not certain that replicating intraalveolar bacteria release sufficiently high concentrations of pneumolysin to produce acute lung injury. Second, pneumolysin is a member of a family of related "thiol-activated" bacterial toxins that are oxygen labile (27), and thus might be inactivated either by oxidizing conditions within the pulmonary alveolus or by hydrogen peroxide generated by the pneumococcus itself. Finally, pneumolysin and other thiol-acti- vated toxins can be inhibited by nanomolar concentrations of cholesterol (17), present in the lung in alveolar surfactant, cellular membranes, and serum lipoprotein. Thus, despite extensive biochemical and molecular characterization of its properties in vitro, the contribution of pneumolysin to the early pathogenesis of pneumococcal pneumonia remained unknown.

Using a technique of direct e.t. instillation of bacterial inocula (23), which delivers a known quantity of bacteria at a discrete time, we were able to quantify accurately changes in bacterial numbers in lung samples at early times after infection. Comparison of infection of Swiss mice with genetically constructed pneumolysin-deficient mutant and wild-type strains of type $2 S$. pneumoniae confirmed that $\operatorname{PLY}(+)$ bacteria were more virulent than PLY $(-)$ pneumococci after both systemic and mucosal infection, as previously described (12). Importantly, we observed that mice had a marked intrinsic resistance to infection by e.t. compared with i.p. route, similar to that noted during infection of BALB/c mice with these same strains (12). These results demonstrate that the mouse lung serves as a major natural line of defense against disseminated pneumococcal infection.

Supporting our observations of lung cell injury by pneumolysin in vitro (7), we demonstrated that pneumolysin released by bacteria during pneumococcal pneumonia in vivo was cytotoxic to the alveolar-capillary barrier. The alveolar epithelium is the principal barrier to colloid and crystalloid flux into the alveolus, and injury to the epithelium permits leakage of serum proteins into the alveoli (28). Infection with PLY (-) bacteria produced minimal increases in concentrations of albumin recovered by alveolar lavage, similar to those seen from instillation of PBS alone. In contrast, infection with PLY $(+)$ bacteria produced substantial increases in alveolar albumin concentrations, indicating that pneumolysin is released in sufficient quantities and remains sufficiently cytotoxic, despite potential inhibitors, to produce acute lung injury in vivo.

Pneumolysin also produced specific pathogenic effects during the early hours of $S$. pneumoniae infection, including increased intraalveolar replication of bacteria, penetration of pneumococci from alveoli into the interstitium of the lung, and dissemination of bacteria from lung into the bloodstream. These effects could not be attributed to an intrinsic defect in growth of the PLY (-) strain, which grew as well as the PLY $(+)$ strain in vitro in a variety of culture media, including homogenized murine lung. Facilitation of dissemination of pneumococci from alveolar to vascular compartments presumably is related to pneumolysin's ability to disrupt the alveolar epithelium and endothelium $(6,7)$, permitting bacterial penetration into and multiplication within the pulmonary interstitium. Pneumococci most likely move from the interstitium into the bloodstream via lymphatic drainage, although direct dissemination into the vascular space following endothelial injury by pneumolysin is also possible. Pneumolysin may also contribute to the earlier and high-grade bacteremia seen with the PLY $(+)$ strain through its ability to inhibit the clearance of pneumococci from the vascular space (12).

Our studies also provide evidence that pneumolysin's cytotoxic and complement-activating properties independently contribute to enhanced bacterial growth during early pneumococcal pneumonia. When inocula of the PLY $(-)$ strain were supplemented with purified recombinant pneumolysins, pneumococcal growth at $3 \mathrm{~h}$ after infection was facilitated by pneumolysin's 
cytolytic activity. However, compared with studies using PLY $(+)$ bacteria, fewer viable PLY $(-)$ bacteria were recovered from lungs at 6 and $24 \mathrm{~h}$ after instillation of pneumococci with added recombinant pneumolysin, despite a similar degree of initial alveolar injury. These disparities may reflect the difference between the addition of a single dose of exogenous pneumolysin to the PLY $(-)$ strain and the ongoing release of pneumolysin in vivo from the PLY $(+)$ strain. The latter may facilitate bacterial growth at 6 and $24 \mathrm{~h}$ by continued alveolar damage, interference with complement-mediated clearance, or other mechanisms not yet delineated.

Flooding of alveoli with serum and erythrocytes following pneumolysin-mediated injury may support intraalveolar replication of bacteria by providing necessary nutrients and antioxidants. Acute hemorrhagic edema may also decrease bacterial elimination (29). Potentially, influx of serum albumin may neutralize intraalveolar free fatty acids, which are the principal antipneumococcal factor in alveolar lining fluids of the murine lung (30). The cytotoxicity of pneumolysin for monocytes (5) and neutrophils (4) in vitro may also increase recovery of viable pneumococci by inhibiting bacterial elimination by these immune cells. However, we observed that the rates of net clearance of pneumococci during the period of neutrophil influx were essentially identical for both the PLY $(+)$ and PLY $(-)$ strains, suggesting that pneumolysin's effect on immune cells makes a minor contribution at most to the early pathogenesis of pneumonia.

Pneumolysin activates the classical complement system in vitro (10). However, this function has not previously been demonstrated to affect pneumococcal growth or elimination in vivo. By supplementing inocula of PLY $(-)$ bacteria with modified recombinant pneumolysins possessing either cytolytic or complement-activating properties, we demonstrated that intact complement-activating activity correlated with enhanced alveolar bacterial growth at $6 \mathrm{~h}$ after e.t. infection. Similarly, numbers of PLY $(-)$ bacteria were increased from 3 to $6 \mathrm{~h}$ in mice with genetic complement deficiency compared with congenic complement-sufficient mice. In both sets of experiments, the net clearance of PLY $(-)$ bacteria from alveolar lavage was predominantly affected. Taken together, these studies suggest that the ability of pneumolysin to activate complement independently contributes to the intraalveolar replication of pneumococci during the early period of neutrophil influx in pneumococcal pneumonia. These observations are consistent with a model of ineffective activation of the complement system by pneumolysin, which may consume complement factors and divert complement opsonins away from bacterial cells.

In summary, we have demonstrated in vivo that pneumolysin's cytotoxic and complement-activating properties contribute independently to facilitate intraalveolar replication and dissemination of pneumococci into lung tissue and the bloodstream during the initial hours of experimental pneumonia. The important role of pneumolysin in the early pathogenesis of pneumococcal pneumonia suggests that immunization against pneumolysin might decrease acute lung injury and bacteremia during pulmonary infection. Neutralizing antibodies to pneumolysin are produced during pneumococcal infection in humans (31, 32 ), and immunization with pneumolysin has been shown to reduce or delay death in animals (14). Furthermore, as a protein toxin produced by essentially all clinical isolates of $S$. pneumo- niae, pneumolysin is a promising candidate for use in a proteinconjugate pneumococcal vaccine (33).

\section{Acknowledgments}

We thank Claire Pomeroy and Dennis Niewoehner for their critical reviews of this manuscript.

This work was supported by the U.S. Department of Veterans Affairs Research Service (grant to E.N. Janoff), National Institutes of Health grants R29-AI34051 (J.B. Rubins) and R29-AI31373 (E.N. Janoff), and the Medical Research Council and the Royal Society (T.J. Mitchell). Dr. Mitchell is a Royal Society Research Fellow.

\section{References}

1. Kramer, M. R., B. Rudensky, I. Hadas-Halperin, M. Isacsohn, and E. Melzer. 1987. Pneumococcal bacteremia-no change in mortality in 30 years: analysis of 104 cases and review of the literature. Isr. J. Med. Sci. 23:174-180.

2. Jetté, L. P. and F. Lamothe. 1989. Surveillance of invasive Streptococcus pneumoniae infection in Quebec, Canada, from 1984 to 1986: serotype distribution, antimicrobial susceptibility, and clinical characteristics. J. Clin. Microbiol. 27:1-5.

3. Musher, D. M. 1992. Infections caused by Streptococcus pneumoniae: clinical spectrum, pathogenesis, immunity, and treatment. Clin. Infect. Dis. 14:801-809.

4. Paton, J. C. and A. Ferrante. 1983. Inhibition of human polymorphonuclear leukocyte respiratory burst, bactericidal activity, and migration by pneumolysin. Infect. Immun. 41:1212-1216.

5. Nandoskar, M., A. Ferrante, E. J. Bates, N. Hurst, and J. C. Paton. 1986 Inhibition of human monocyte respiratory burst, degranulation, phospholipid methylation and bactericidal activity by pneumolysin. Immunology. 59:515-520.

6. Rubins, J. B., P. G. Duane, D. Charboneau, and E. N. Janoff. 1992. Toxicity of pneumolysin to pulmonary endothelial cells in vitro. Infect. Immun. 60:17401746.

7. Rubins, J. B., P. G. Duane, D. Clawson, D. Charboneau, J. Young, and D. E. Niewoehner. 1993. Toxicity of pneumolysin to pulmonary alveolar epithelial cells. Infect. Immun. 61:1352-1358.

8. Steinfort, C. R. Wilson, T. Mitchell, C. Feldman, A. Rutman, H. Todd, D. Sykes, J. Walker, K. Saunders, P. W. Andrew, G. J. Boulnois, and P. J. Cole. 1989. Effect of Streptococcus pneumoniae on human respiratory epithelium in vitro. Infect. Immun. 57:2006-2013.

9. Feldman, C., N. C. Munro, P. K. Jeffery, T. J. Mitchell, P. W. Andrew, G. J. Boulnois, D. Guerreiro, J. A. L. Rohde, H. C. Todd, P. J. Cole, and R. Wilson. 1991. Pneumolysin induces the salient histologic features of pneumococcal infection in the rat lung in vivo. Am. J. Respir. Cell Mol. Biol. 5:416-423.

10. Paton, J. C., B. Rowan-Kelly, and A. Ferrante. 1984. Activation of human complement by the pneumococcal toxin pneumolysin. Infect. Immun. 43:10851087.

11. Houldsworth, S., P. W. Andrew, and T. J. Mitchell. 1994. Pneumolysin stimulates production of tumor necrosis factor alpha and interleukin-1 $\beta$ by human mononuclear phagocytes. Infect. Immun. 62:1501-1503.

12. Berry, A. M., J. Yother, D. E. Briles, D. Hansman, and J. C. Paton. 1989. Reduced virulence of a defined pneumolysin-negative mutant of Streptococcus pneumoniae. Infect. Immun. 57:2037-2042.

13. Berry, A. M., J. C. Paton, and D. Hansman. 1992. Effect of insertional inactivation of the genes encoding pneumolysin and autolysin on the virulence of Streptococcus pneumoniae type 3. Microb. Pathogen. 12:87-93.

14. Paton, J. C., R. A. Lock, and D. J. Hansman. 1983. Effect of immunization with pneumolysin on survival time of mice challenged with Streptococcus pneumoniae. Infect. Immun. 40:548-552.

15. Lock, R. A., D. Hansman, and J. C. Paton. 1992. Comparative efficacy of autolysin and pneumolysin as immunogens protecting mice against infection by Streptococcus pneumoniae. Microb. Pathogen. 12:137-143.

16. Duane, P. G., J. B. Rubins, H. R. Weisel, and E. N. Janoff. 1993. Identification of hydrogen peroxide as a Streptococcus pneumoniae toxin for rat alveolar epithelial cells. Infect. Immun. 61:4392-4397.

17. Johnson, M. K., C. Geoffroy, and J. E. Alouf. 1980. Binding of cholesterol by sulf hydryl-activated cytolysins. Infect. Immun. 27:97-101.

18. Rubins, J. B. and M. R. Freiberg. 1994. Anti-pneumolysin activity of commercially available $\alpha_{1}$-antitrypsin is due to cholesterol impurities. Microb. Pathogen. 16:221-228.

19. Toews, G. B., and W. C. Vial. 1984. The role of C5 in polymorphonuclear leukocyte recruitment in response to Streptococcus pneumoniae. Am. Rev. Resp. Dis. 129:82-86.

20. Saunders, F. K., T. J. Mitchell, J. A. Walker, P. W. Andrew, and G. J. 
Boulnois. 1989. Pneumolysin, the thiol-activated toxin of Streptococcus pneumoniae, does not require a thiol group for in vitro activity. Infect. Immun. 57:25472552.

21. Mitchell, T. J., P. W. Andrew, F. K. Saunders, A. N. Smith, and G. J. Boulnois. 1991. Complement activation and antibody binding by pneumolysin via a region of the toxin homologous to a human acute phase protein. $\mathrm{Mol}$. Microbiol. 5:1883-1888.

22. Mitchell, T. J., J. A. Walker, F. K. Saunders, P. W. Andrew, and G. J. Boulnois. 1989. Expression of the pneumolysin gene in Escherichia coli: rapid purification and biological properties. Biochim. Biophys. Acta. 1007:67-72.

23. Esposito, A. L., and J. E. Pennington. 1983. Effects of aging on antibacterial mechanisms in experimental pneumonia. Am. Rev. Resp. Dis. 128:662-667.

24. Reed, L. J. and H. Muench. 1938. A simple method of estimating fifty per cent endpoints. Am. J. Hygiene. 27:493-497.

25. Wood, W. B. J. 1941. Studies on the mechanism of recovery in pneumococcal pneumonia: I. The action of type specific antibody upon the pulmonary lesion of experimental pneumonia. J. Exp. Med. 73:201-222.

26. Winkelstein, J. A. 1984. Complement and the host's defense against the pneumococcus. Crit. Rev. Microbiol. 11:187-208.

27. Boulnois, G. J., J. C. Paton, T. J. Mitchell, and P. W. Andrew. 1991 Structure and function of pneumolysin, the multifunctional, thiol-activated toxin of Streptococcus pneumoniae. Mol. Microbiol. 5:2611-2616.
28. Wangensteen, O. D., L. E. Wittmers, Jr., and J. A. Johnson. 1969. Permeability of the mammalian blood-gas barrier and its components. Am. J. Physiol. 216:719-727.

29. Johanson, W. G., Jr., S. J. Jay, and A. K. Pierce. 1974. Bacterial growth in vivo: an important determinant of the pulmonary clearance of Diplococcus pneumoniae in rats. J. Clin. Invest. 53:1320-1325.

30. Coonrod, J. D., R. L. Lester, and L. C. Hsu. 1984. Characterization of the extracellular bactericidal factors of rat alveolar lining material. J. Clin. Invest. 74:1269-1279.

31. Leinonen, M., H. Syrjala, E. Jalonen, P. Kujala, and E. Herva. 1990. Demonstration of pneumolysin antibodies in circulating immune complexes-a new diagnostic method for pneumococcal pneumonia. Sero. Immun. Infect. Dis. 4:451 458.

32. Oker-Blom, N. 1948. On antipneumolysin in serum particularly in pneumococcal infections and on its relation to antistreptolysin. Acta Pathol. Microbiol. Scand. Suppl. 72:1-72.

33. Paton, J. C., R. A. Lock, C. J. Lee, J. P. Li, A. M. Berry, T. J. Mitchell, P. W. Andrew, D. Hansman, and G. J. Boulnois. 1991. Purification and immunogenicity of genetically obtained pneumolysin toxoids and their conjugation to Streptococcus pneumoniae type 19F polysaccharide. Infect. Immun. 59:22972304. 